302 - The Effectiveness and Safety of Electroconvulsive Therapy for Treatment Refractory Agitation or Aggression in Major Neurocognitive Disorder

Simon Woo, MD, FRCPC ${ }^{a, b}$; Peter Chan, MD, FRCPC ${ }^{a, c}$; Robyn E Waxman, MD, FRCPC ${ }^{\text {, e, f }}$; Sarah Elmi, MD, FRCPC $^{f}$, Mafalda Musacchio, MD, FRCPC ${ }^{a, g}$; Michael Wilkins-Ho, MD, FRCPC ${ }^{a, c}$; Claire Docherty, MB BCh $\mathrm{BAO}^{\mathrm{a}}$

${ }^{\mathrm{a} D e p a r t m e n t}$ of Psychiatry, University of British Columbia; ${ }^{\mathrm{b}}$ Department of Psychiatry, Royal Columbian Hospital; 'Department of Psychiatry, Vancouver General Hospital;

${ }^{\mathrm{d} D e p a r t m e n t}$ of Psychiatry, University of Toronto; ${ }^{\mathrm{e} C e n t r e}$ for Addiction and Mental Health; ${ }^{\mathrm{f} O n t a r i o}$ Shores Centre for Mental Health Sciences; ${ }^{\text {g}}$ Parkview Tertiary Intensive Older Adult Mental Health Unit

\title{
Introduction:
}

Fifty to ninety percent of individuals with Major Neurocognitive Disorder (MNCD) have Neuropsychiatric Symptoms (NPS) ${ }^{1}$. Agitation and aggression are amongst the most persistent and treatment-refractory symptom clusters. Patients with these NPS are associated with increased risk of institutionalization, psychotropic medication use, caregiver burden, and mortality ${ }^{2}$.

Safe and effective treatments for NPS are lacking. Consensus guidelines emphasize the initial use of non-pharmacologic approaches though supportive evidence is limited ${ }^{3}$.

Extensive research has established the safety and efficacy of ECT in elderly patients with depression and other psychiatric conditions ${ }^{6}$. Clinical experience suggests that ECT is a valuable treatment option in MNCD-related treatment refractory NPS cases ${ }^{7-10}$. However, data supporting the efficacy and safety of this practice is scant.

\section{Materials and Method:}

Patients admitted to the geriatric psychiatry inpatient units who meet the inclusion criteria, were recruited from 2 Vancouver sites and 3 unit at Ontario Shores. These patients had an anesthesia consultation to evaluate their safety of going through ECT. Consent was obtained from their substitute decision makers. All patients enrolled are already on psychotropic medications.

Inclusion criteria:

* diagnosis of dementia according to DSM-IV-TR criteria

* Failed non-pharmacological treatment and 2 failed trials of psychotropic medications

* referred to ECT specifically for treatment of agitation and aggression

Data collection:

* Validated scales were administered at baseline, at regular intervals during ECT treatment, and up to 8 weeks after completion of the ECT course

* The Neuropsychiatric Inventory (NPI) and Pittsburgh Agitation Scale (PAS) were the main measure of agitation and aggression.

The final number recruited to the study was 29 patients and mean age was 74 .

\section{Results}

